\title{
The Structure of the Molecular Envelope around CRL 2688
}

\author{
I. Yamamura ${ }^{1,2}$, S. Deguchi ${ }^{3}$ and T. Kasuga ${ }^{4}$ \\ ${ }^{1}$ Institute of Astronomy, University of Tokyo; \\ ${ }^{2}$ SRON Laboratory for Space Research, Groningen; ${ }^{3}$ Nobeyama Radio Observatory, Japan; \\ ${ }^{4}$ Hosei University, Japan
}

We have observed CRL 2688 (the Egg nebula) in the ${ }^{13} \mathrm{CO} J=1-0$ and CS $J=1-0$ and $2-1$ lines by the Nobeyama Millimeter Array with a resolution of about 4 ". The ${ }^{13} \mathrm{CO}$ velocity channel maps show that emission consists of three components; a spherical core, an extended envelope, and a bipolar high-velocity component (Yamamura et al. 1995).A spherical shape of the core despite of the maximum optical depth of about the unity indicates that the disk-like structure, expected from the shape of the bipolar nebula, is smaller than the present beam size (corresponding to about $6 \times 10^{16} \mathrm{~cm}$ at $1 \mathrm{kpc}$ ). The combined maps made from the data by the NMA and the Nobeyama 45-m telescope show that emission spreads towards the south of the center more then the opposite direction.

The three-dimensional density distribution in the envelope was calculated based on the combined maps (Yamamura et al. 1996). It turned out that the mass-loss rate of the object increased from about $3000 \mathrm{yr}$ ago and reached the maximum value of about $3 \times$ $10^{-4} \mathrm{M}_{\odot} \mathrm{yr}^{-1}$, then rapidly decreased about $200 \mathrm{yr}$ ago.

The CS $J=1-0$ maps show the rather spherical envelope, while the $J=2-1$ maps exhibits two bright spots (Kasuga et al. 1996). It is very interesting that the direction of the line connecting the two spots is consistent with the direction of the high-velocity flow, and inclined about $30-60^{\circ}$ from the bipolar axis of the reflection nebula. A simple modelcalculations show that these bright spots can be interpreted by the enhancement of the CS abundance due to shock chemistry.

I.Y. is supported by the JSPS Research Fellowships for Young Scientists.

\section{REFERENCES}

Yamamura, I. et al. 1995, ApJ, 439, L13.

Yamamura, I. et al. 1996, ApJ, 465, 926.

Kasuga, T., Yamamura, I., \& Deguchi, S. 1996, A\&A, in press 

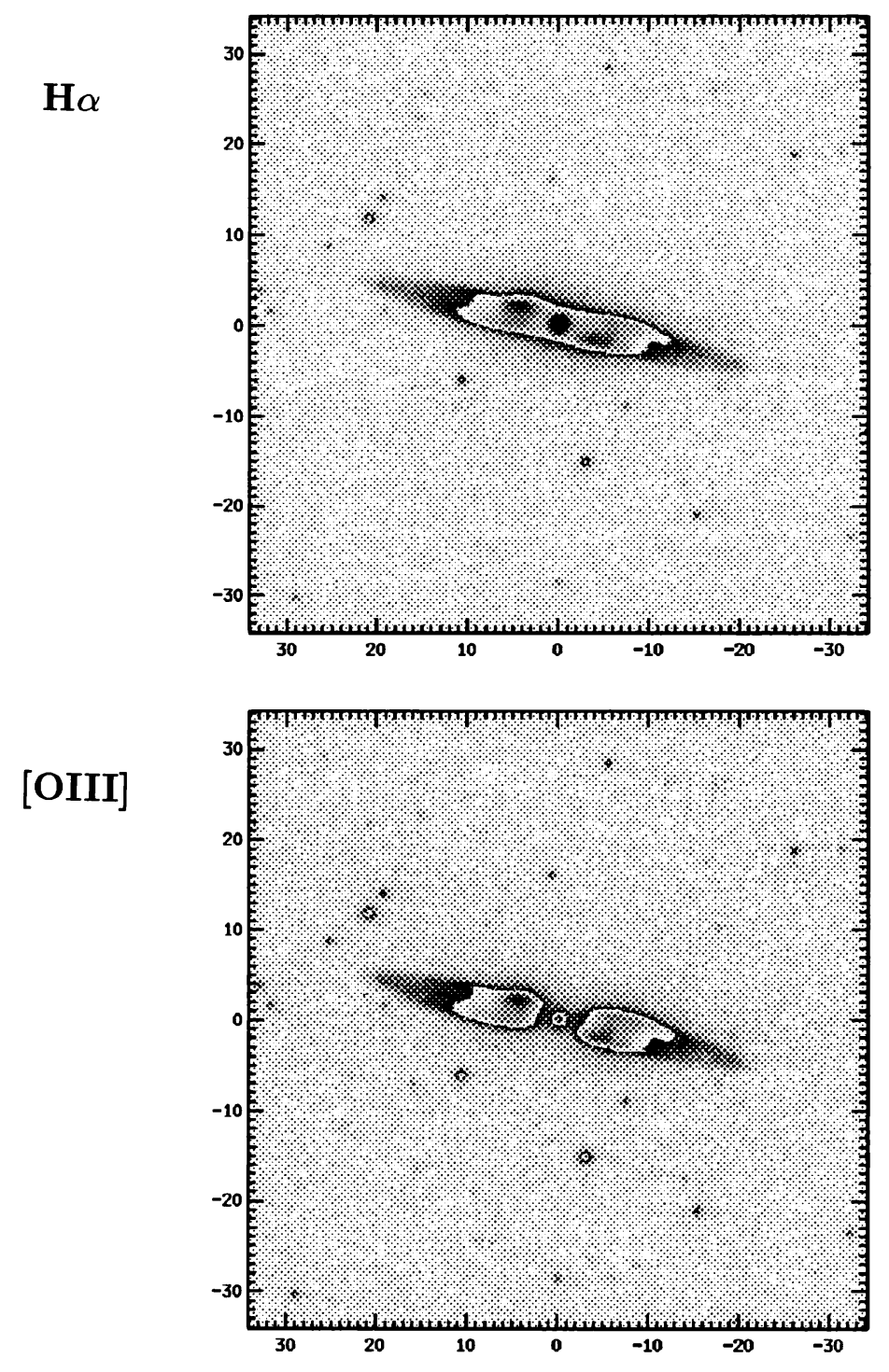

He 2-437 061.3+03.6

From: "The IAC Morphological Catalog of Northern Galactic Planetary Nebulae", A. Manchado, M.A. Guerrero, L. Stanghellini, M. Serre-Ricart.

courtesy: A. Manchado 